\title{
Annexin A10 is involved in the induction of pancreatic duodenal homeobox-1 in gastric cancer tissue, cells and organoids
}

\author{
AKIRA ISHIKAWA $^{1}$, NAOYA SAKAMOTO ${ }^{1}$, RIRINO HONMA ${ }^{1}$, DAIKI TANIYAMA ${ }^{1}$, \\ KAHO FUKADA $^{1}$, TAKUYA HATTORI ${ }^{1}$, KAZUHIRO SENTANI ${ }^{1}$, NAOHIDE OUE ${ }^{1}$, \\ KAZUYOSHI YANAGIHARA ${ }^{2}$, KAZUAKI TANABE ${ }^{3}$, HIDEKI OHDAN $^{3}$ and WATARU YASUI $^{1}$ \\ ${ }^{1}$ Department of Molecular Pathology, Institute of Biomedical and Health Sciences, Hiroshima University, \\ Hiroshima 734-8551; ${ }^{2}$ Division of Biomarker Discovery, Exploratory Oncology Research and Clinical Trial \\ Center, National Cancer Center, Tokyo 277-8577; ${ }^{3}$ Department of Gastroenterological and Transplant Surgery, \\ Applied Life Sciences, Institute of Biomedical and Health Sciences, Hiroshima University, Hiroshima 734-8551, Japan
}

Received January 23, 2019; Accepted October 17, 2019

DOI: $10.3892 /$ or.2019.7422

\begin{abstract}
Gastric cancer (GC) is the third most common cause of cancer-related death in the world. Annexin A10 (ANXA10), a member of the Annexin family, is a calcium-/phospholipid-binding protein; however, little is known concerning its functions. It is still unclear what molecule is involved in the induction of ANXA10. In the present study, we performed immunohistochemistry to evaluate the expression of ANXA10, pancreatic and duodenal homeobox-1 (PDX1) and mucin phenotype markers in 130 GC samples. ANXA10 was detected in $63(48 \%)$ of the $130 \mathrm{GC}$ cases and loss of ANXA10 was significantly correlated with disease progression and poor clinical outcomes in GC. PDX1 was significantly correlated with ANXA10 in GC cases and cell lines. Although PDX1 was not significantly correlated with the GC cases with any of the mucin phenotypes, ANXA10 was preferentially detected in the GC cases with the gastric mucin phenotype. As a further investigation, we generated organoids derived from human GC and identified the duplication of the mucin phenotypes of GC by immunohistochemistry. The repression effect on cell growth that was observed in the ANXA10-knockdown cell lines was also clearly observed in the human gastric organoids. We demonstrated that the expression of ANXA10 was correlated with the gastric mucin
\end{abstract}

Correspondence to: Professor Wataru Yasui, Department of Molecular Pathology, Institute of Biomedical and Health Sciences, Hiroshima University, 1-2-3 Kasumi, Minami-ku, Hiroshima 734-8551, Japan

E-mail: wyasui@hiroshima-u.ac.jp

Abbreviations: GC, gastric cancer; ANXA10, annexin A10; PDX1, pancreatic and duodenal homeobox-1; IHC, immunohistochemistry; RT-qPCR, quantitative RT-PCR

Key words: annexin A10, pancreatic and duodenal homeobox-1, organoid, mucin phenotype, gastric cancer phenotype and ANXA10 was involved in the induction of PDX1 expression in GC. We also provided evidence that GC organoids represent a powerful tool for scrutinizing the biology of GC, especially with regard to the mucin phenotype.

\section{Introduction}

Gastric cancer (GC) is the third leading cause of cancer-related death in the world (1). A deeper understanding of the pathogenesis and biological features of GC is necessary to further enhance early detection and treatment methods. The identification of novel biomarkers, which could become both potent new tools for the diagnosis of GC and targets for treatment, is one of the main strategies for improving the diagnosis and treatment of GC (2). In addition to the Lauren histology-based classification (3), GC can be subdivided into four phenotypes according to mucin expression (2). Accumulating evidence suggests that the gastric/intestinal mucin phenotypes of GC have different clinical characteristics and specific genetic and epigenetic changes (4). Thus, the classification of the subtype of GC is important for clinical decision-making.

Annexin A10 (ANXA10), a calcium-/phospholipid-binding protein, is a member of the Annexin family. It has been reported that Annexin family proteins play important roles in calcium signaling, cell motility, differentiation and proliferation $(5,6)$. Expression of ANXA10 is observed in foveolar and glandular cells of the normal antral or the body-type gastric mucosa in the stomach $(7,8)$. Other normal organs, including the duodenum and urothelial epithelium, also show the robust expression of ANXA10 (8). With regard to cancer tissue, the expression of ANXA10 has been observed in several types of cancer, including GCs, but the clinical significance of ANXA10 is still controversial in various types of cancer (8-13). In colorectal adenocarcinomas, several studies that have confirmed the induction of the expression of gastric mucins in serrated neoplasms have shown that the aberrant expression of ANXA10 is significantly correlated with gastric differentiation (14-17).

The expression of ANXA10 is frequently found to be downregulated in GC, and its downregulation is associated 
with a poor prognosis (18). However, the role of the expression of ANXA10 in tumor progression depends on the morphologic classification of GC; in diffuse-type GC, the expression of ANXA10 is associated with better survival, whereas in intestinal-type GC, the expression of ANXA10 is associated with a poorer prognosis (in comparison to the lack of expression of ANXA10) (7). With regard to biological functions, a small number of studies have reported that ANXA10 plays a tumor-suppressive role through the regulation of cellular proliferation and apoptosis in GC cell lines $(18,19)$. Taken together with these data, ANXA10 is more likely to be involved in the differentiation of the stomach and it is presumed that ANXA10 basically acts as a tumor suppressor as long as the cancer cells retain the expression of ANXA10. However, no studies have reported a correlation between the expression of ANXA10 and the mucin phenotype of GC, and only a few studies have described the functional role of ANXA10 in GC.

Pancreatic and duodenal homeobox-1 (PDX1) is one of the transcription factors that belongs to the homeobox gene family, which is important in the differentiation and development of the antrum of the stomach, duodenum and pancreas (20). In the adult, the expression of PDX1 is selectively observed in pancreatic $\beta$ cells, Brunner's glands of the duodenum and pyloric glands of the stomach. In cancerous tissues, PDX1 is aberrantly expressed in ductal adenocarcinoma of the pancreas (21) and functions as a tumor suppressor in GC (22). In addition, we recently found that PDX1 is frequently expressed in serrated adenocarcinoma of the colon and ANXA10 is also a valuable diagnostic marker for serrated adenocarcinoma of the colon (23). Although aberrant expression of PDX1 has been reported mainly in cancers of the digestive organs and somehow seems to have a role in the pathogenesis of carcinomas, it has not been irrefutably ascertained how the expression of PDX1 is regulated.

In the present study, we examined the expression and distribution of ANXA10 in GC by immunohistochemistry (IHC), and investigated the relationship between the expression of ANXA10 and the gastric/intestinal mucin phenotypes of GC in order to test the abovementioned hypothesis. Furthermore, we also aimed to identify the effect of ANXA10 on the expression of other molecules, especially focusing on the molecules related to the differentiation of the gastrointestinal organs. Among these molecules, it was decided to focus on PDX1. In order to analyze the relationship between ANXA10 and PDX1 in GC tissues and the effect of the deregulation of ANXA10 on the expression of PDX1, both GC cell lines and organoids were used since organoids may be useful to reveal the correlation between ANXA10 and PDX1 in the minimum unit of glands from non-cancerous and GC tissue, which could potentially elucidate the actual correlation of these two molecules in vivo.

\section{Materials and methods}

Tissue samples. Samples from 130 primary tumors that had been collected from patients diagnosed with $\mathrm{GC}$ who underwent surgery at Hiroshima University Hospital (Hiroshima, Japan) between 2003 and 2007 (range of age, 38-82 years; male, $n=82$; female, $n=48$ ) were retrospectively analyzed. The patients were followed up by their physicians until their death or the date of the last documented contact. For the immunohistochemical analyses (IHC), archival formalin-fixed, paraffin-embedded tissues from $130 \mathrm{GC}$ patients who had undergone surgical excision as treatment for GC were consecutively collected. One representative tumor block from each patient, including the tumor center, invasive front, and the tumor-associated non-neoplastic mucosa, was evaluated by IHC. Tumor staging was determined according to the TNM classification system. The histological classifications were determined based on the guidelines of the Japanese Research Society for Gastric Cancer. Written informed consent for the establishment of organoids was obtained from all of the patients. This study was approved by the Ethics Committee for Human Genome Research of Hiroshima University, Hiroshima (E-597-01). This study was conducted in accordance with the Ethical Guidance for Human Genome/Gene Research of the Japanese Government.

IHC. IHC was performed with a Dako EnVision+ mouse peroxidase detection system (cat. no. K5007; DakoCytomation). Antigen retrieval was performed by heating citrate buffer (pH 6.0) in a microwave for $30 \mathrm{~min}$. Peroxidase activity was blocked with $3 \% \mathrm{H}_{2} \mathrm{O}_{2}$-methanol for $10 \mathrm{~min}$, and sections were incubated with normal goat serum (cat. no. X090710; DakoCytomation) for 20 min to block nonspecific antibody binding sites. Sections were incubated with a rabbit monoclonal anti-ANXA10 antibody (dilution 1:1,000, cat. no. NBP1-90156; Novus Biologicals) or a rabbit monoclonal anti-PDX1 antibody (dilution 1:1,000, cat. no. ab134150; Abcam) for $1 \mathrm{~h}$ at room temperature, followed by incubation with EnVision+ anti-rabbit peroxidase for $1 \mathrm{~h}$. For a color reaction, sections were incubated with the DAB substrate-chromogen solution (cat. no. K3468; DakoCytomation) for $10 \mathrm{~min}$. Sections were counterstained with $0.1 \%$ hematoxylin. Negative controls were created by omission of the primary antibody.

The expression of ANXA10 was scored in all tumors as positive or negative. When $>10 \%$ of tumor cells were stained, the immunostaining was considered positive for ANXA10. Using these definitions, 3 surgical pathologists (AI, NS and DT), with no knowledge of the clinical and pathologic parameters or the outcomes of the patients, independently reviewed the immunoreactivity of each specimen. Interobserver differences were resolved by consensus review at a double-headed microscope after an independent review.

Sample collection from the database. We downloaded all of the GC transcriptome profiles and clinical data from The Cancer Genome Atlas (TCGA) database (https://xenabrowser.net/dat apages/?cohort=TCGA\%20Stomach\%20Cancer\%20(STAD)\& removeHub=https $\% 3 \mathrm{~A} \% 2 \mathrm{~F} \% 2 \mathrm{Fx}$ ena.treehouse.gi.ucsc.edu $\% 3$ A443). Up until September 2018, the public database included the expression profiles of $375 \mathrm{GC}$ tissues and 32 normal tissues obtained by RNA-seq (level 3). Our research conformed to the guidelines published in TCGA. We took the logarithm of the expression difference between normal and tumor samples.

Phenotypic analysis. GCs were classified into 4 phenotypes: Gastric (G), intestinal (I), gastric and intestinal mixed (GI) and null $(\mathrm{N})$ types according to the mucin expression pattern. To analyze the phenotypic expression of GC, we performed IHC (as described above) with 4 antibodies, all from Novocastra 
Laboratories, Inc.: Anti-MUC5AC (NCL-MUC-5AC), anti-MUC6 (NCL-MUC-6), anti-MUC2 (PA0155) and anti-CD10 (NCL-L-CD10-270). The criteria for the classification of G- and I-type GCs have been described previously (2).

Cell lines. Five cell lines derived from human GC (MKN-1, MKN-7, MKN-45, MKN-74 and HSC-57) were used. MKN1, MKN7,MKN45 and MKN74 were purchased from the Japanese Collection of Research Bioresources Cell Bank (Osaka, Japan) and HSC-57 was established by Dr Kazuyoshi Yanagihara (Exploratory Oncology Research and Clinical Trial Center, National Cancer Center) (24). All cell lines were maintained in RPMI-1640 medium (cat. no. 05918; Nissui Pharmaceutical Co., Ltd.) containing $10 \%$ fetal bovine serum (cat. no. 14-501F; BioWhittaker) in a humidified atmosphere of $5 \% \mathrm{CO}_{2}$ and $95 \%$ air at $37^{\circ} \mathrm{C}$.

Western blotting. Western blotting was performed as described previously (25). Immunocomplexes were visualized with an Amersham ECL Western blot detection system (cat. no. RPN2109; GE Healthcare). $\beta$-actin (cat. no. A5316; Sigma-Aldrich; Merck KGaA) was also stained as a loading control.

Lentiviral and retroviral vectors. For constitutive expression of human ANXA10, cDNA was PCR amplified from MKN-74 cells and subcloned into pDON5neo (Takara Bio) using a retrovirus vector with psPAX2 envelope and pMD2.G packaging plasmids, according to the manufacturer's instructions.

pTRIPZ-short hairpin (sh)ANXA10 (cat. no. 11199; GE Healthcare) was constructed as follows: sh-ANXA10-1, TTG CTGATTAGATAGTAGG; sh-ANXA10-2, TAATTGCTG ATTAGATAGT; sh-ANXA10-3, TAATTGCTGATTAGA TAGT. ANXA10 was amplified from MKN-74 cDNA by a PCR. The PCR fragment was ligated into a pDON-5 Neo plasmid (cat. no. 3655; Takara) at the BamHI sites. All of the above were cloned into the lentiviral transfer vector pTRIPZ (cat. no. RHS4740-EG11199; GE Healthcare) and were verified by sequencing. A lentivirus was produced by cotransfecting the transfer vector containing the gene of interest, the VSVG envelope glycoprotein, and the lentiviral packaging vector, into 293T cells (\#HCL4517; Dharmacon). The virus supernatant was concentrated by ultrafiltration using Millex-HV (Millipore). In parallel, transduced cells were selected in $3.0 \mu \mathrm{g} / \mathrm{ml}$ puromycin (cat. no. P7255; Sigma-Aldrich; Merck KGaA) or $250 \mu \mathrm{g} / \mathrm{ml} \mathrm{G} 418$ (cat. no. 11811031; Life Technologies)-containing media, corresponding to the minimum time taken for untransduced cells to completely die in the selection media. Protein knockdown or overexpression was verified by western blotting.

Cell growth assay. To examine cell growth, an MTT assay was performed as described previously (26). Cell growth was monitored after 1, 2, 4 and 8 days, and OD570 was used for the detection of absorbance.

Organoid culture and viral infection of organoids. Organoids were derived from the stomach of patients who underwent gastrectomy. Gastric organoids were generated and propagated using the previously described 'TMDU protocol' (27), with minor modifications. The stomach and tumor lesions were removed, minced into small pieces, and suspended in $5 \mathrm{ml}$ of DMEM (Thermo Fisher Scientific, Inc.) supplemented with $100 \mathrm{U} / \mathrm{ml}$ penicillin (Thermo Fisher Scientific, Inc.), $100 \mathrm{mg} / \mathrm{ml}$ streptomycin (Thermo Fisher Scientific, Inc.), $50 \mathrm{mg} / \mathrm{ml}$ gentamicin (Thermo Fisher Scientific, Inc.) and $1 \%$ FBS (complete DMEM), to which 15 mM EDTA was added. The mixture was shaken for $1 \mathrm{~h}$ at $4^{\circ} \mathrm{C}$. The released crypts were washed extensively, pelleted, and resuspended in $200 \mu \mathrm{l}$ of the Matrigel (Corning, Inc.) and placed in 3 wells of 24-well plates. After polymerization, $500 \mu \mathrm{l}$ of 'stomach' and 'colon' media were added to each well to produce the respective organoids. The details of ingredients in each medium are summarized in Table SI. The medium was changed every 2-3 days. The organoids were split at a ratio of 1:3 every 7-8 days. Previously described protocols (with minor modifications) were used for the gene knockdown and forced-expression studies (28).

$R T-q P C R$. Total RNA was isolated from frozen samples or cancer cell lines using Isogen (Nippon Gene), and $1 \mu \mathrm{g}$ of total RNA was converted to cDNA with a First Strand cDNA Synthesis Kit (GE Healthcare). Real-time detection of the emission intensity of SYBR Green bound to double-stranded DNA was performed with a CFX Connect Real-Time System (Bio-Rad Laboratories). The thermocycling conditions consisted of denaturation at $95^{\circ} \mathrm{C}$ for $10 \mathrm{sec}$ and annealing at $60^{\circ} \mathrm{C}$ for $30 \mathrm{sec}$ repeated for 40 cycle. $A C T B$-specific PCR products, which were amplified from the same RNA samples, served as internal controls. Relative quantification was determined using the $\Delta \Delta \mathrm{Cq}$ method (29). The primer sequences are summarized in Table SII.

Statistical analysis. Correlations between clinicopathological parameters/markers of mucin phenotype and ANXA10 expression were analyzed by Chi-square test, Fisher's exact test and two-sided test. Kaplan-Meier survival curves were constructed for ANXA10-positive or ANXA10-negative patients. Differences between survival curves were tested for statistical significance using a log-rank test. Expression levels of ANXA10 and PDX1 were analyzed by Student's t-test. The data concerning cell growth assay was also analyzed by Tukey's test or Student's t-test. The data obtained from TCGA was analyzed by Spearman's Rho as for the correlation between ANXA10 and PDX1 expression.

\section{Results}

Expression and the clinicopathological significance of ANXA10 in GC. We analyzed the expression of ANXA10 in $130 \mathrm{GC}$ cases by immunohistochemical staining. In the non-neoplastic gastric mucosa, the expression of ANXA10 was observed on the nucleoli of the foveolar cells (Fig. 1A). No expression of ANXA10 was observed on epithelial cells with intestinal metaplasia (Fig. 1B). In GC tissue, the robust nuclear staining of ANXA10 was detected in both the well-differentiated (Fig. 1C) and poorly differentiated (Fig. 1D) types of GC. Since the median value of the positivity of the GC cells in the sections was $\sim 9.6 \%$, the GC cases with staining of $>10 \%$ of tumor cells were regarded as positive cases. IHC 

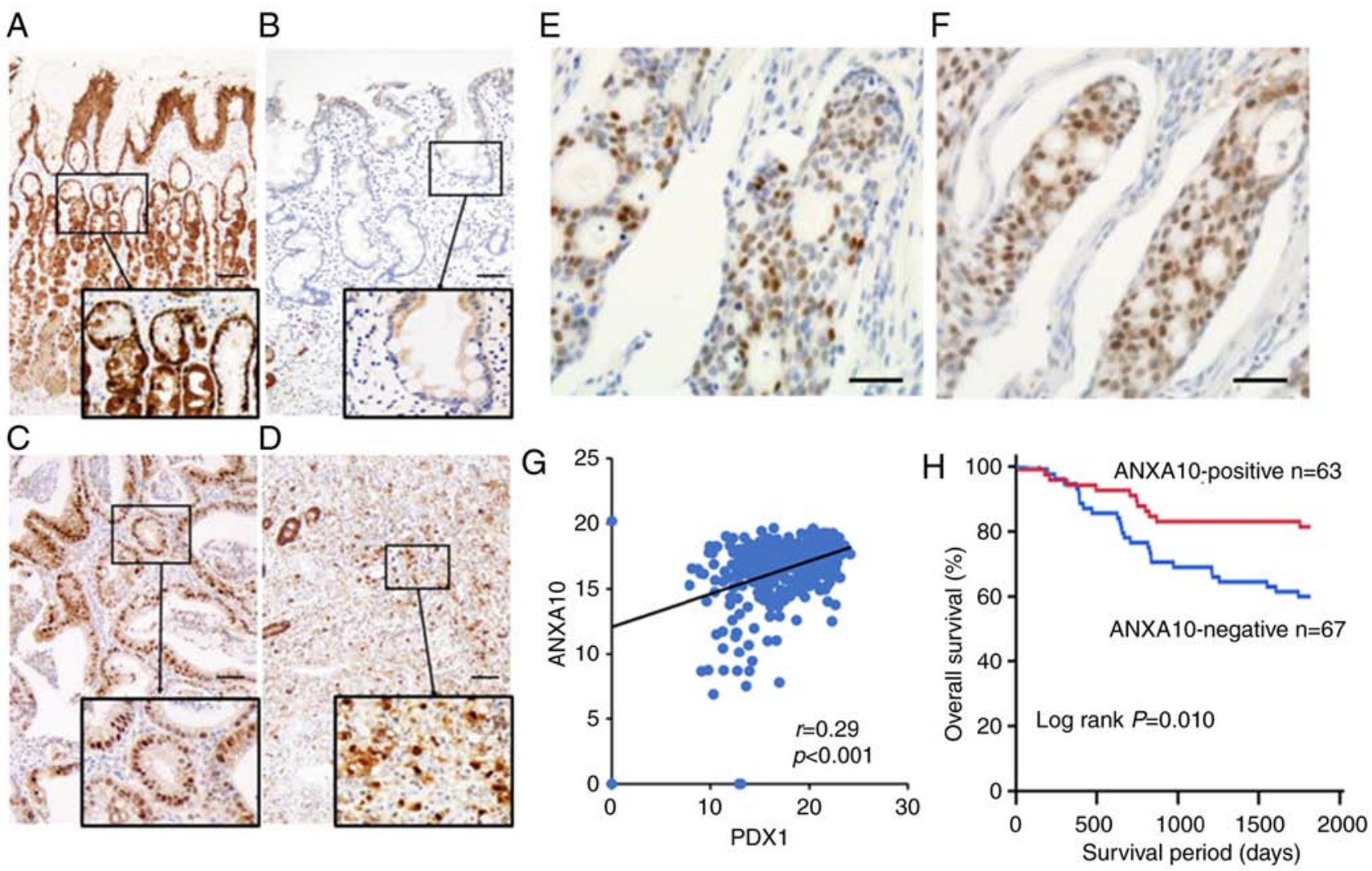

Figure 1. Expression of ANXA10 in GC. (A) Immunohistochemical staining of ANXA10 in normal gastric glands. The nucleoli of normal cells of the gastric mucosa were positive for ANXA10. Original magnification, x100; scale bars, $100 \mu \mathrm{m}$. (B) Immunohistochemical staining of ANXA10 in intestinal metaplasia. (C and D) Immunohistochemical staining of ANXA10 in well-differentiated type (C) and poorly differentiated type (D) GC. Original magnification, x100; scale bars, $100 \mu \mathrm{m}$. Staining of the nucleoli revealed ANXA10 positivity (E and F) Expression of ANXA10 (E) and PDX1 (F) in a GC patient. Original magnification, x400; scale bars, $100 \mu \mathrm{m}$. (G) Correlation between ANXA10 and PDX1 expression in the GC dataset in TCGA. (H) A Kaplan-Meier plot of survival based on ANXA10 expression in the group of 130 GC patients. GC, gastric cancer; ANXA10, annexin A10; PDX1, pancreatic and duodenal homeobox-1; TCGA, The Cancer Genome Atlas.

staining of PDX1 was conducted for specimens from $130 \mathrm{GC}$ cases; the expression of PDX1 was detected in the nucleoli of GC cells (Fig. 1F) along with the expression of ANXA10 (Fig. 1E). Correlations were analyzed between the expression of ANXA10 and the clinicopathological characteristics of the GC cases. A loss of ANXA10 expression was associated with $\mathrm{pN}$ stage $(\mathrm{P}=0.020)$, $\mathrm{pM}$ stage $(\mathrm{P}<0.001)$ and $\mathrm{pStage}$ $(\mathrm{P}=0.008)$ (Table I). The association between ANXA10 and PDX1 expression in GC was also investigated as previously expression of both ANXA10 and PDX1 was frequently found in serrated adenocarcinoma of the colon (23). We found that the expression of ANXA10 was significantly associated with that of PDX1 in GC cases $(\mathrm{P}=0.016)$ (Table I). The correlation between ANXA10 and PDX1 was also determined in the cohort that was uploaded in the TCGA. A significant correlation was found between them ( $r=0.29, \mathrm{P}<0.001$; Fig. 1G). Kaplan-Meier analysis showed that a loss of ANXA10 expression was significantly associated with poor survival in GC patients $(\mathrm{P}=0.010$; Fig. $1 \mathrm{H})$. These results suggest that ANXA10 plays an important role in the progression of GC.

Association of ANXA10/PDX1 with mucin phenotypes of $G C$. The comparison between ANXA10 and markers of the mucin phenotype revealed that ANXA10 was more frequently expressed in $44(66 \%)$ of the 67 MUC5AC-positive GC cases than in $19(30 \%)$ of the 63 MUC5AC-negative
GC cases, and that it was less frequently expressed in $3(19 \%)$ of the 16 CD10-positive GC cases than in $60(53 \%)$ of the 114 CD10-negative GC cases (Table II). Representative expression patterns of ANXA10 and other mucin markers are shown in Fig. S1. We used these 4 markers to phenotypically classify the GC cases as follows: G-type $(n=53 ; 41 \%)$, GI-type $(n=20 ; 15 \%)$, I-type $(n=25 ; 19 \%)$ and N-type $(n=32$; 25\%). The expression of ANXA10 was more frequently observed in G-type GC in comparison to the other types of GC (GI-, I- and N-type) (P<0.001, Table II). We also examined the correlation between PDX1 and markers of the mucin phenotype as PDX1 is a transcription factor that plays critical roles in the histogenesis of the stomach (19). In the comparison, neither of the markers of mucin phenotype showed significant correlation with the expression of PDX1 in the GC cases (Table III). Taken together with these findings, only ANXA10 was more likely to be expressed in GC cases with a gastric mucin phenotype and PDX1 did not show any preferences in GC cases with the various specific mucin phenotypes.

Effects of ANXA10 on the expression of PDX1 in GC. Although our results indicated that ANAXA10 and PDX1 were preferentially co-expressed in GC tissues, it remained unclear whether there is an interaction between ANAX10 and PDX1. We examined the expression of ANXA10 and 
Table I. Association between ANXA10 expression and clinicopathological characteristics of the patients with GC $(\mathrm{N}=130)$.

\begin{tabular}{|c|c|c|c|}
\hline \multirow[b]{2}{*}{ Characteristics } & \multicolumn{2}{|c|}{ Annexin A10 expression } & \multirow[b]{2}{*}{ P-value } \\
\hline & Positive (\%) & Negative & \\
\hline Age (years) & & & 0.074 \\
\hline$<65$ & $39(56)$ & 31 & \\
\hline$\geq 65$ & $24(40)$ & 36 & \\
\hline Sex & & & 0.174 \\
\hline Male & $36(44)$ & 46 & \\
\hline Female & $27(56)$ & 21 & \\
\hline pT stage & & & 0.084 \\
\hline pT1 & $33(57)$ & 25 & \\
\hline $\mathrm{pT} 2 / 3 / 4$ & $30(42)$ & 42 & \\
\hline pN stage & & & 0.020 \\
\hline pN0 & $41(58)$ & 30 & \\
\hline $\mathrm{pN} 1 / 2 / 3$ & $22(37)$ & 37 & \\
\hline pM stage & & & $<0.001$ \\
\hline $\mathrm{pM0}$ & $59(57)$ & 44 & \\
\hline pM1 & $4(15)$ & 23 & \\
\hline pStage & & & 0.008 \\
\hline pStage I & $40(60)$ & 27 & \\
\hline pStage II/III/IV & $23(37)$ & 40 & \\
\hline Histology & & & 0.112 \\
\hline Intestinal-type & $26(41)$ & 37 & \\
\hline Diffuse-type & $37(55)$ & 30 & \\
\hline \multicolumn{4}{|l|}{ PDX1 } \\
\hline Positive & $45(57)$ & 34 & 0.016 \\
\hline Negative & $18(35)$ & 33 & \\
\hline
\end{tabular}

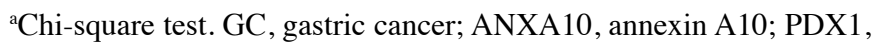
pancreatic and duodenal homeobox-1.

PDX1 by western blotting using $5 \mathrm{GC}$ cell lines. The level of PDX1 expression was undetectable in 1 cell line (MKN-1); however, the expression of PDX1 was not detectable in any of the GC cell lines in which the expression of ANXA10 was undetectable (Fig. 2A). We also evaluated the expression of ANXA10 and PDX1 by RT-qPCR, and similar expression patterns were found as those for the western blot analysis (Fig. 2B and C). We examined the transition of ANXA10 expression using MKN-74 and MKN-45 cells transfected with ANXA10-specific shRNAs. All of the 3 different shRNAs (sh-ANXA10-1-3) repressed the expression of ANXA10 to some extent; accordingly, the expression of PDX1 was decreased in the MKN-74 cells (Fig. 2D) and MKN-45 cells (Fig. 2E). To further investigate the direct correlation between ANXA10 and PDX1, HSC-57 cells were stably transfected with pDON-ANXA10. HSC-57-ANXA10 expressed ANXA10 as expected and provided a significantly higher level of PDX1 in comparison to the HSC-57-empty vector cells (Fig. 2F). We also performed an MTT assay in order to examine the effect of ANXA10 on cell growth. The cell growth of MKN-74-shRNA cells was significantly increased in comparison to the MKN-74-scramble cells from day 2 (Fig. 2G). The same trend was observed in the MKN-45 cells (Fig. 2H). Conversely, the cell growth of HSC-57-ANXA10 cells was significantly inhibited in comparison to the HSC-57-empty scramble cells on day 4 and 6 (Fig. 2I). We also examined the effect of overexpression of ANXA10 on the expression of the markers for mucin phenotype using HSC-57-ANXA10 cells, and found that expression of any of the markers was not affected by ANXA10 forced-expression (Fig. S2). These results demonstrated that ANXA10 could play an important role in the regulation of PDX1 expression in $\mathrm{GC}$ cells.

Establishment of GC organoids with the gastric and intestinal mucin phenotypes. In order to further validate the relationship between ANXA10 and PDX1, GC organoids that displayed a distinct mucin phenotype were established. Pieces of the GC specimens were divided into two and then cultured in stomach and colon media, respectively. We successfully established GC organoids from 10 patients, and the mucin phenotypes varied with each case (Fig. 3 and Table SIII). It is noteworthy that we successfully generated an organoid from scirrhous-type GC in case 1, which was characterized by diffuse/scattered clusters of cancer cells and abundant stroma. While the primary tumor did not show any glandular structures, the organoids somehow formed glands that were composed by cancer cells with a high nucleus/cytoplasm ratio; akin to the cancer cells of the primary tumor.

Effect of ANXA10 on the PDX1 expression in GC patient-derived organoids. To further verify the tight correlation between ANXA10 and PDX1, we used organoids derived from the non-neoplastic epithelium and GC tissue. Robust expression of ANXA10 and PDX1 was found in the organoids from the non-neoplastic mucosa and GC showing only the gastric mucin phenotype (Fig. 4A and B), which was consistent with the data obtained from the immunohistochemical analysis using GC samples. We investigated the transition of the ANXA10 and PDX1 expression by IHC staining using the abovementioned organoids transfected with ANXA10 shRNAs (shANXA10) in order to ascertain whether similar changes would be observed in the organoids as those in the GC cell lines. In both organoids generated from GC tissue and the non-neoplastic epithelium, the PDX1 expression was significantly repressed by ANXA10 knockdown (Fig. 4A-C). In comparison to the GC organoids transfected with shANXA10, the morphology of the organoids transfected with shANXA10 was dramatically altered: Every single component looked thin and monotonous with a high nuclear-cytoplasmic ratio, which indicated that these cells could potentially be stem/progenitor-like cells. Taken together, the results suggest that organoids are likely to be useful for revealing dynamic molecular alterations in specific organs, and our results further suggest that ANXA10 is involved in the regulation of the expression of PDX1 in both normal stomach epithelium and GC and that ANXA10 could also play an essential role in the differentiation of the normal gastric epithelium. 
Table II. Expression of ANXA10 and markers of the mucin phenotype in GC/mucin phenotypes of GC (N=130).

\begin{tabular}{|c|c|c|c|c|}
\hline \multirow[b]{2}{*}{ Markers } & \multicolumn{2}{|c|}{ ANXA10 expression } & \multirow[b]{2}{*}{ P-value ${ }^{a}$} & \multirow[b]{2}{*}{ P-value } \\
\hline & Positive (\%) & Negative & & \\
\hline \multicolumn{5}{|l|}{ Gastric markers } \\
\hline MUC5AC & & & $<0.001$ & $<0.05$ \\
\hline Positive & $44(66)$ & 23 & & \\
\hline Negative & $19(30)$ & 44 & & \\
\hline MUC6 & & & 0.485 & NS \\
\hline Positive & $6(40)$ & 9 & & \\
\hline Negative & $57(50)$ & 58 & & \\
\hline \multicolumn{5}{|l|}{ Intestinal markers } \\
\hline MUC2 & & & 0.836 & NS \\
\hline Positive & $15(47)$ & 17 & & \\
\hline Negative & $48(49)$ & 50 & & \\
\hline CD10 & & & 0.011 & NS \\
\hline Positive & $3(19)$ & 13 & & \\
\hline \multirow[t]{2}{*}{ Negative } & $60(53)$ & 54 & & \\
\hline & \multicolumn{2}{|c|}{ ANXA10 expression } & \multirow{2}{*}{\multicolumn{2}{|c|}{ P-value ${ }^{c}$ (G-types vs. others) }} \\
\hline Mucin phenotypes & Positive (\%) & Negative & & \\
\hline G-type & $34(51)$ & $19(30)$ & \multirow{4}{*}{\multicolumn{2}{|c|}{$<0.001$}} \\
\hline GI-type & $12(18)$ & $8(13)$ & & \\
\hline I-type & $5(7)$ & $20(32)$ & & \\
\hline N-type & $16(24)$ & $16(25)$ & & \\
\hline
\end{tabular}

${ }^{a}$ Chi-square test. ${ }^{b}$ Two-sided test. ${ }^{c}$ Fisher's exact test. NS, not significant. GC, gastric cancer; ANXA10, annexin A10.

Table III. Expression of PDX1 and markers of the mucin-phenotype in GC $(\mathrm{N}=130)$.

\begin{tabular}{lccc}
\hline & \multicolumn{2}{c}{ PDX1 expression } & \\
\cline { 2 - 3 } Markers & Positive (\%) & Negative & P-value $^{\mathrm{a}}$ \\
\hline Gastric markers & & & \\
MUC5AC & $39(58)$ & 28 & \\
Positive & $40(63)$ & 23 & \\
Negative & $7(46)$ & 8 & 0.234 \\
MUC6 & $72(62)$ & 43 & \\
Positive & & & \\
Negative & & & \\
Intestinal markers & $19(59)$ & 13 & \\
MUC2 & $60(61)$ & 38 & \\
Positive & & & \\
Negative & $10(62)$ & 6 & \\
CD10 & $69(60)$ & 45 & \\
Positive & & & \\
Negative & & & \\
\hline
\end{tabular}

${ }^{a}$ Chi-square test. GC, gastric cancer. PDX1, pancreatic and duodenal homeobox-1.

\section{Discussion}

We immunohistochemically assessed the expression of annexin A10 (ANXA10) in gastric cancer (GC) and confirmed the clinicopathological significance and functional roles of ANXA10 in GC using GC cell lines, tissues and patient-derived organoids. One of the novel findings of this study is that ANXA10 regulates the expression of pancreatic and duodenal homeobox-1 (PDX1); this was verified in both GC cell lines and organoids. PDX1 plays an important role in the differentiation and development of the pancreas, duodenum and antrum (20) and is regarded as a tumor suppressor (22); both of these roles are quite similar to the roles of ANXA10. In light of the expression pattern revealed by IHC, previous studies have observed the expression of ANXA10 (8) and PDX1 (30) in GC, and the PDX1 was localized in nucleoli as well as ANXA10, supporting the correlation between these two molecules. While we indicated various relationships between ANXA10 and PDX1, it was not ascertained whether ANAX10 regulates PDX1 in a direct or indirect manner. One challenge in resolving this issue is that the functional role of ANXA10 remains unclear; there is no solid evidence of a key regulator or direct target of ANXA10. Additional studies, perhaps using a proteome analysis, will likely be needed to advance our understanding 

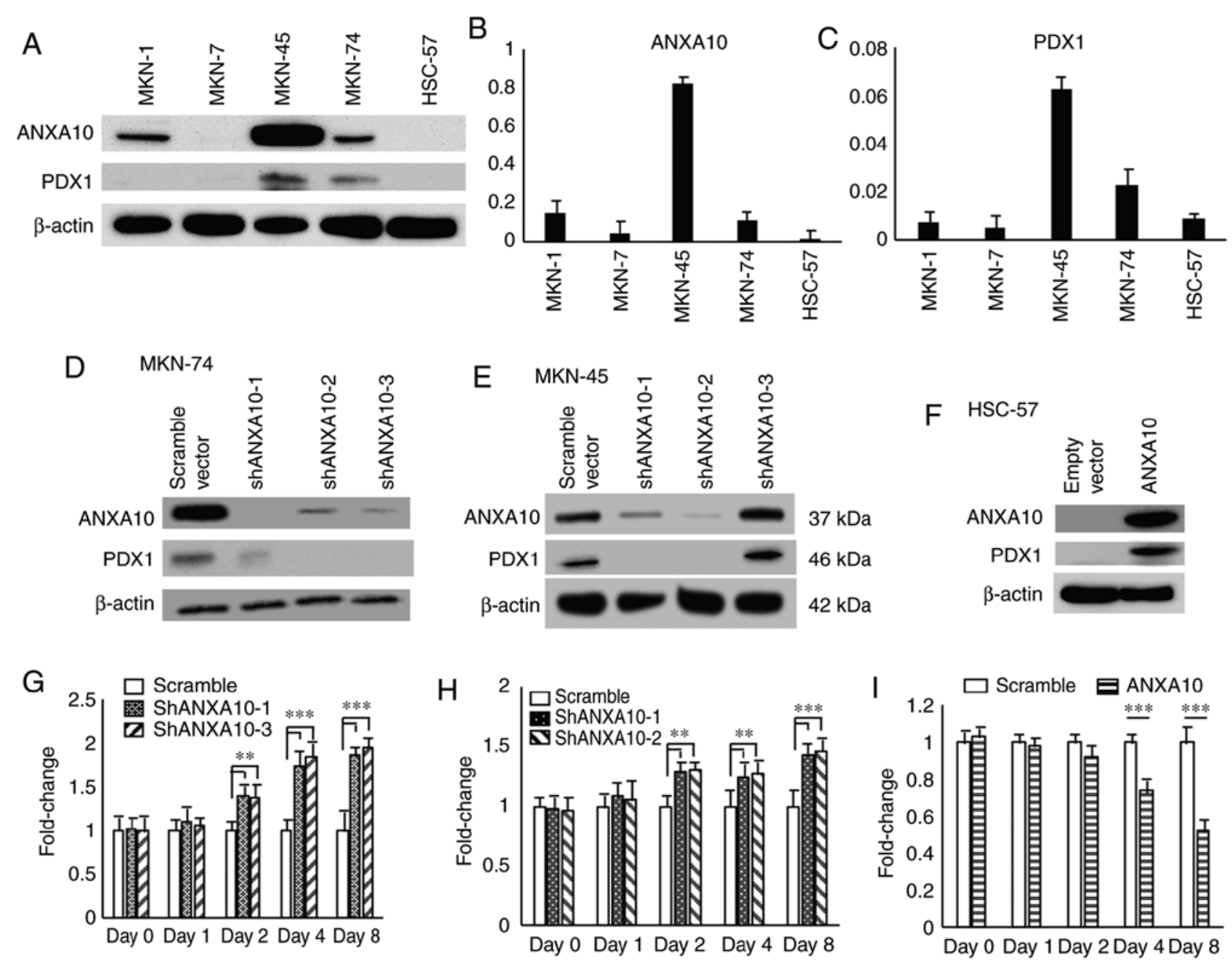

Figure 2. Effects of ANXA10 on the expression of PDX1. (A) Western blot analysis of ANXA10 and PDX1 proteins in 5 GC cell lines. (B and C) RT-qPCR for (B) ANXA10 and (C) RT-qPCR for PDX1 expression in 5 GC cell lines. Bars and error bars represent the mean and SD, respectively, of 3 independent experiments. (D and E) Western blot analysis of the ANXA10 and PDX1 proteins in (D) MKN-74 and (E) MKN-45 cells transfected with ANXA10 shRNA (shANXA10-1-3) or scramble vector. The $\beta$-actin levels were measured as a loading control. (F) Western blot analysis of ANXA10 and PDX1 proteins in HSC-57 cells transfected with ANXA10 expression vector or empty vector. (G and H) Cell proliferation assays of (G) MKN-74 and (H) MKN-45 cells transfected with ANXA10 shRNA vector or scramble vector. Cell growth was assessed by MTT assays at 1,2,4 and 8 days after seeding on 96-well plates. Bars and error bars represent the mean and SD, respectively, of 3 independent experiments $\left.{ }^{* *} \mathrm{P}<0.01,{ }^{* * * *} \mathrm{P}<0.001\right)$. (I) Cell proliferation assay of HSC-57 cells transfected with ANXA10 expression vector or empty vector $\left({ }^{* * * *} \mathrm{P}<0.001\right)$. GC, gastric cancer; ANXA10, annexin A10; PDX1, pancreatic and duodenal homeobox-1.

of the mechanism involved in the regulation of ANXA10 and its target, which could potentially reveal the fundamental mechanisms through which ANXA10 contributes to the progression of GC.

As for the functional role of ANXA10 in GC, it was verified that ANXA10 basically plays a tumor-suppressive role in GC, which is consistent with the data in previous studies $(18,19)$. We observed expression of ANXA10 in normal stomach tissues and loss of the expression of ANXA10 in some of the GC tissues, most of which showed advanced clinicopathological features and/or poor clinical outcome. In contrast, previous studies described that normal squamous epithelium usually does not show expression of ANXA10 and some squamous cell carcinoma tissues inversely exhibited robust expression of ANXA10, which was found to significantly contribute to the progression of squamous cell carcinoma $(9,10)$. These data indicate that the functional role of ANXA10 in human cancers has a bilateral function displaying tumor-suppressive and promotive roles depending on the original organs. Further studies, such as constructing a library of the expression pattern of ANXA10 in human normal and cancer tissues, would be instrumental in revealing the entire aspect of the variety of the functional roles of ANXA10, which could potentially lead to a breakthrough in the functional role of ANXA10 in human tissues. Another limitation of our study is that we were not able to further validate our data concerning the functional role of ANXA10 in an in vivo study. These considerations point to the current issues concerning the functional role of ANXA10 that could be further examined.

In the present study, we aimed to ascertain whether overexpression of ANXA10 directly affects the mucin phenotypes of GC cells using HSC-57-ANXA10 cells as shown in Fig. 2. However, the cells did not show any differences regardless of the successful overexpression of ANXA10 and subsequent PDX1 expression. One of the reasons is that HSC-57 cells have been differentiated into an intestinal mucin phenotype with expression of CDX2, which is a representative transcription factor for the organogenesis and maintenance of 


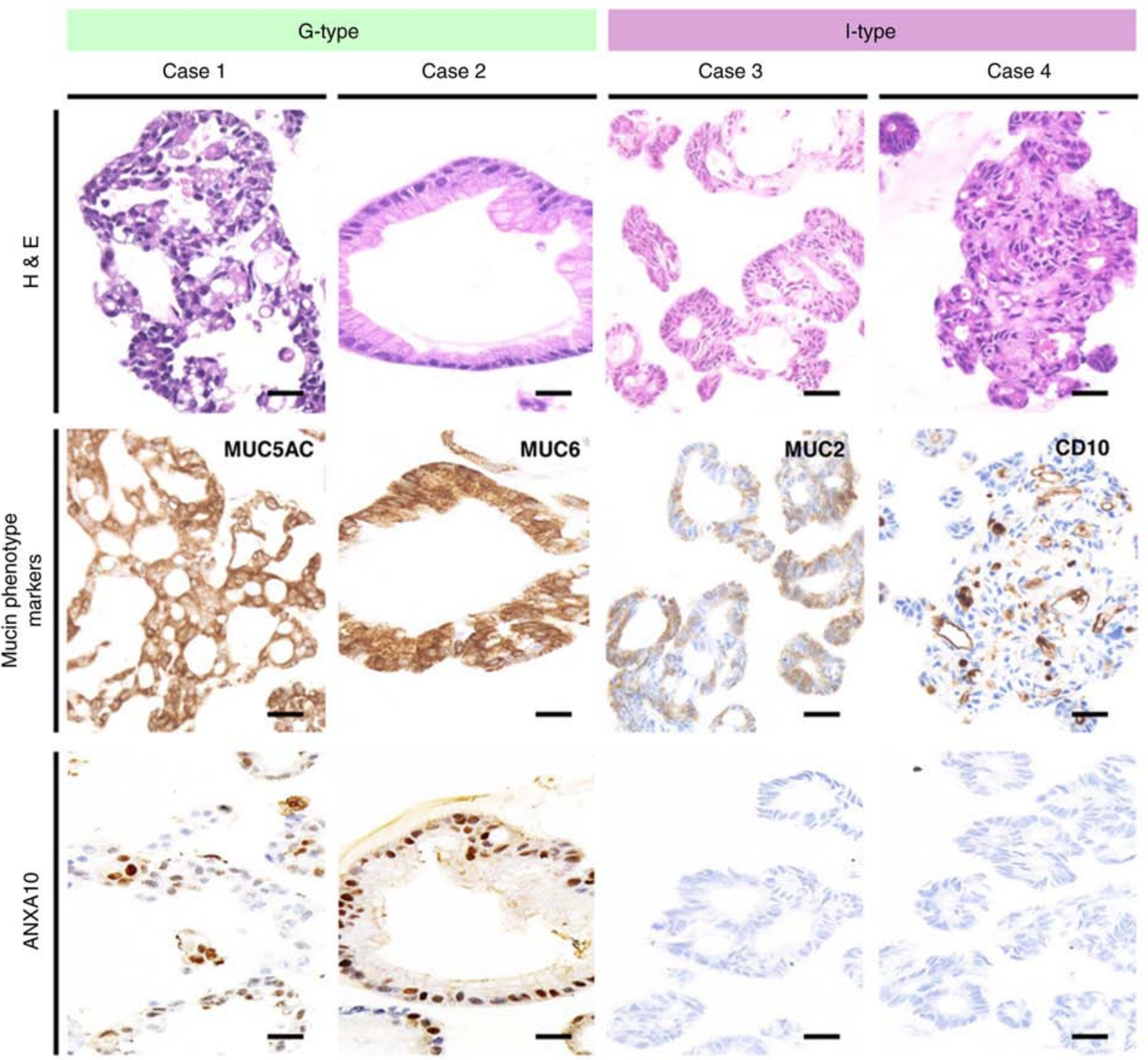

Figure 3. Establishment of GC organoids and the immunohistochemical analysis of the mucin-phenotype and the expression of ANXA10. Original magnification, x400; scale bars, $50 \mu \mathrm{m}$. GC, gastric cancer; ANXA10, annexin A10; H\&E, hematoxylin and eosin staining.

the small intestine and colon. We hypothesized that GC cells with $\mathrm{N}$-type could be an ideal model as they are more likely to represent an undifferentiated status of GC, but unfortunately no GC cells with N-type were currently available. Further studies using the models that can recapitulate undifferentiated status of GC, such as GC cells and organoids with $\mathrm{N}$-type, could provide more solid evidence concerning the functional roles of ANXA10 in regards to mucin phenotypes of GC.

An epoch-making result of the present study is that we successfully established a GC organoid library. Organoid culturing is a novel 3D stem cell culture system that has been developed by exploiting the understanding of stem cell niche factors (31). These organoids reproduce the histopathological grade and differentiation capacity of their primary tumor (32). Only one study has reported the establishment of GC organoids from surgically resected samples (33); however, that study only mentioned that the GC organoid represented the p53 mutation. In the present study, we described a novel method for recapitulating the mucin phenotypes of primary GC in each GC organoid, which was quite useful for validating the specific expression and function of ANXA10 in G-type GC. The mucin phenotype of GC has mainly been examined by pathologists, and it has been regarded as just one of the sub-classifications of GC. Recent evidence suggests that GCs should be classified into 4 groups based on data from comprehensive characterization by whole-genome sequencing (34). When examining the representative characteristics of each group proposed by TCGA, we realized that the 'Microsatellite instability (MSI) group' and GCs with gastric mucin phenotype share many genetic and phenotypic features, including MLH1 silencing and hypermutations in tumor-suppressor genes. In addition, a similar trend was observed between the 'Chromosomal instability (CIN) group' and GCs with the intestinal mucin phenotype, which is characterized by a high frequency of p53 mutations $(2,34)$. Taking this information into account, it is believed that our GC organoids, which exhibit a distinct mucin phenotype, will be highly valuable for scrutinizing GC biology based on the TCGA classification. Moreover, 

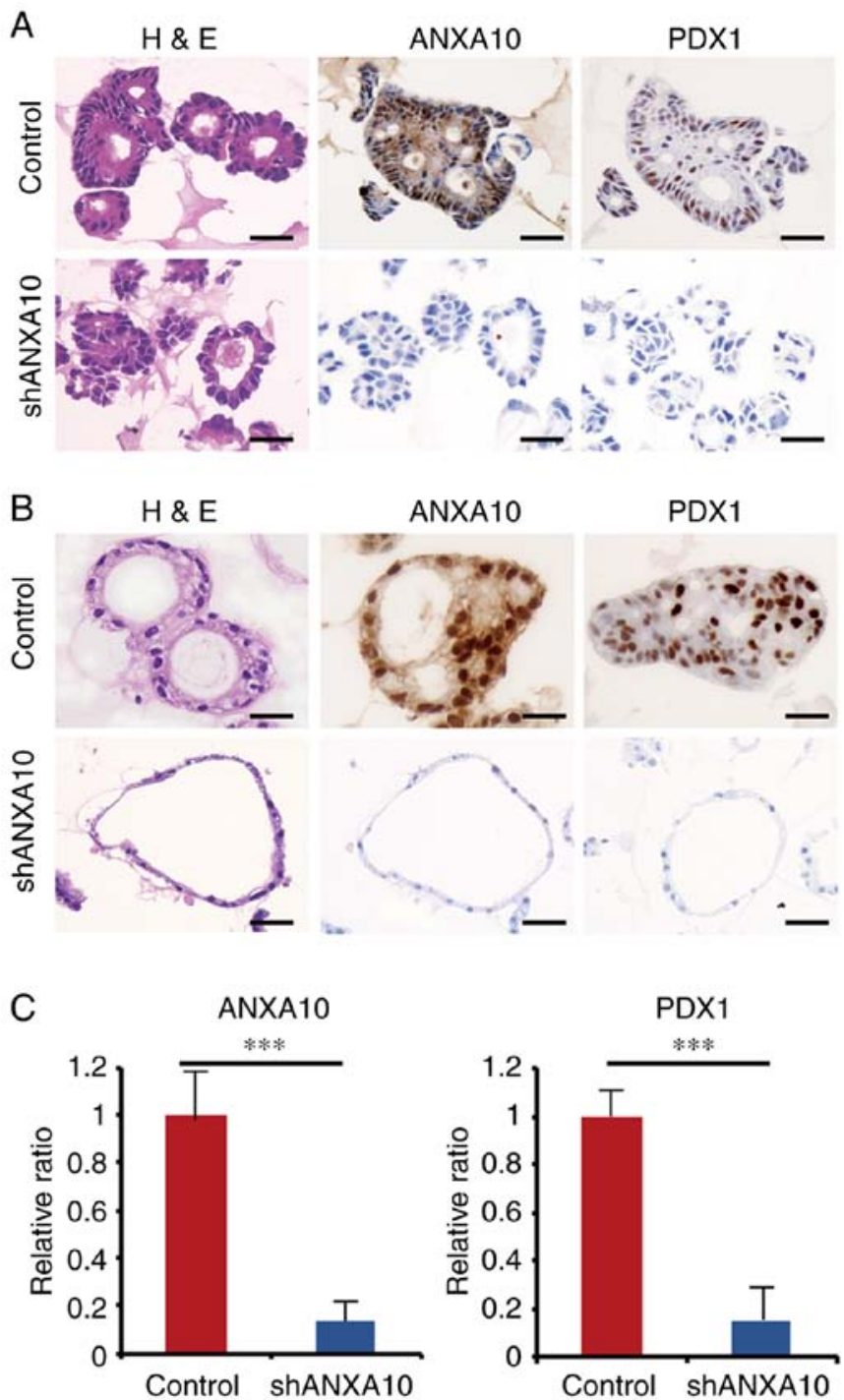

Figure 4. (A) Expression of ANXA10 and PDX1 in patient-derived GC organoids transfected with ANXA10 shRNA vector (shANXA10) or scramble vector. Original magnification, $\mathrm{x} 400$; scale bars, $50 \mu \mathrm{m}$. (B) Expression of ANXA10 and PDX1 in patient-derived normal gastric organoids transfected with ANXA10 shRNA vector (shANXA10) or scramble vector. Original magnification, $\mathrm{x} 400$; scale bars, $50 \mu \mathrm{m}$. (C) RT-qPCR for ANXA10 and PDX1 in patient-derived GC organoids transfected with ANXA10 shRNA vector (shANXA10) or scramble vector. Bars and error bars represent the mean and SD, respectively, of 3 independent experiments $\left({ }^{* * * *} \mathrm{P}<0.001\right)$. GC, gastric cancer; ANXA10, annexin A10; PDX1, pancreatic and duodenal homeobox-1; H\&E, hematoxylin and eosin staining.

another cutting-edge use for organoid libraries is for drug screening. van de Wetering et al reported a screening strategy using a colorectal cancer organoid library (35). Taken together, our GC organoid library is considered to have great potential for investigating drug sensitivity and/or resistance, as well as the daisy chain-like genetic and phenotypic features of GC, and could possibly be a powerful platform for establishing 'personalized-medicine' in GC treatment.

In conclusion, we demonstrated the clinical significance of ANXA10 in GC. We also disclosed that ANXA10 is involved in the regulation of PDX1; a finding that was verified in GC tissues, cell lines and organoids. In addition, we highlighted the usefulness of GC organoids in the detailed study of the mucin phenotype of GC. Both longitudinal and cross-sectional studies using our organoid library could potentially yield valuable information on various issues concerning the pathogenesis and clinical features of GCs.

\section{Acknowledgements}

We thank Mr. Shinichi Norimura (Technical Center, Hiroshima University) for his excellent technical assistance. This research was carried out with the kind cooperation of the Research Center for Molecular Medicine of the Faculty of Medicine of Hiroshima University. We also thank the Analysis Center of Life Science of Hiroshima University for the use of their facilities. The R-spondin-producing cell line was a kind gift from Professor Jeffery Whitsett (Cincinnati Children's Hospital Medical Center, Cincinnati, OH, USA). We would like to thank Professor Eric Fearon (University of Michigan, Ann Arbor, MI, USA) for providing collaborative research resources and comments.

\section{Funding}

The present study was supported by Grants-in-Aid for Scientific Research (JP15H04713, JP16K08691, JP16H06999) and for Challenging Exploratory Research (26670175, JP16K15247) from the Japan Society for the Promotion of Science.

\section{Availability of data and materials}

All data generated or analyzed during this study are included either in this article or in the supplementary information files.

\section{Authors' contributions}

NS designed the study. KS, NO, KT and HO collected and analyzed the patient clinical data. AI, RH, DT, KF and TH performed the experiments and collected and analyzed the data. NS KS, NO, KY, KT, HO and WY interpreted and analyzed the results. AI, NS and WY drafted and edited the manuscript. All authors read and approved the manuscript and agree to be accountable for all aspects of the research in ensuring that the accuracy or integrity of any part of the work are appropriately investigated and resolved.

\section{Ethics approval and consent to participate}

The Institutional Review Board of Hiroshima University Hospital approved the present study (IRB\# E-597-01). Appropriate written informed consent was obtained from each patient. The present study was conducted in accordance with the Ethical Guidance for Human Genome/Gene Research of the Japanese Government.

\section{Patient consent for publication}

Not applicable.

\section{Competing interests}

The authors declare that they have no competing interests. 


\section{Authors' information}

Profssor Wataru Yasui: ORCID: orcid.org/0000-00028647-8405.

\section{References}

1. Ferlay J, Soerjomataram I, Dikshit R, Eser S, Mathers C, Rebelo M, Parkin DM, Forman D and Bray F: Cancer incidence and mortality worldwide: Sources, methods and major patterns in GLOBOCAN 2012. Int J Cancer 136: E359-E386, 2015.

2. Oue N, Sentani K, Sakamoto N and Yasui W: Clinicopathologic and molecular characteristics of gastric cancer showing gastric and intestinal mucin phenotype. Cancer Sci 106 951-958, 2015.

3. Lauren P: The two histological main types of gastric carcinoma: Diffuse and so-called intestinal-type carcinoma. An attempt at a histo-clinical classification. Acta Pathol Microbiol Scand 64 31-49, 1965.

4. Endoh Y, Sakata K, Tamura G, Ohmura K, Ajioka Y, Watanabe H and Motoyama T: Cellular phenotypes of differentiated-type adenocarcinomas and precancerous lesions of the stomach are dependent on the genetic pathways. J Pathol 191: 257-263, 2000.

5. Ohnishi M, Tokuda M, Masaki T, Fujimura T, Tai Y, Matsui H, Itano T, Ishida T, Takahara J and Konishi R: Changes in annexin I and II levels during the postnatal development of rat pancreatic islets. J Cell Sci 107: 2117-2125, 1994.

6. Gerke V and Moss SE: Annexins: From structure to function. Physiol Rev 82: 331-371, 2002

7. Lu SH, Chen YL, Shun CT, Lai JN, Peng SY, Lai PL and Hsu HC: Expression and prognostic significance of gastric-specific annexin A10 in diffuse- and intestinal-type gastric carcinoma. J Gastroenterol Hepatol 26: 90-97, 2011

8. Lu SH, Yuan RH, Chen YL, Hsu HC and Jeng YM: Annexin A10 is an immunohistochemical marker for adenocarcinoma of the upper gastrointestinal tract and pancreatobiliary system. Histopathology 63: 640-648, 2013.

9. Kodaira H, Koma YI, Hosono M, Higashino N, Suemune K, Nishio M, Shigeoka M and Yokozaki H: ANAX10 induction by interaction with tumor-associated macrophages the growth of esophageal squamous cell carcinoma. Pathol Int 69: 135-147, 2019.

10. Shimizu T, Kasamatsu A, Yamamoto A, Koike K, Ishige S, Takatori H, Sakamoto Y, Ogawara K, Shiiba M, Tanzawa H and Uzawa K: Annexin A10 in human oral cancer: Biomarker for tumoral growth via G1/S transition by targeting MAPK signaling pathways. PLoS One 7: e45510, 2012.

11. Masaki T, Tokuda M, Ohnishi M, Watanabe S, Fujimura T, Miyamoto K, Itano T, Matsui $\mathrm{H}$, Arima K, Shirai M, et al: Enhanced expression of the protein kinase substrate annexin in human hepatocellular carcinoma. Hepatology 24: 72-81, 1996.

12. Kim JH, Rhee YY, Kim KJ, Cho NY, Lee HS and Kang GH: Annexin A10 expression correlates with serrated pathway features in colorectal carcinoma with microsatellite instability. APMIS 122: 1187-1195, 2014.

13. Tsai JH, Lin YL, Cheng YC, Chen CC, Lin LI, Tseng LH, Cheng ML, Liau JY and Jeng YM: Aberrant expression of annexin A10 is closely related to gastric phenotype in serrated pathway to colorectal carcinoma. Mod Pathol 28: 268-278, 2015.

14. Gonzalo DH, Lai KK, Shadrach B, Goldblum JR, Bennett AE, Downs-Kelly E, Liu X, Henricks W, Patil DT, Carver P, et al: Gene expression profiling of serrated polyps identifies annexin A10 as a marker of a sessile serrated adenoma/polyp J Pathol 230: 420-429, 2013.

15. Bartley AN, Thompson PA, Buckmeier JA, Kepler CY, Hsu CH, Snyder MS, Lance P, Bhattacharyya A and Hamilton SR: Expression of gastric pyloric mucin, MUC6, in colorectal serrated polyps. Mod Pathol 23: 169-176, 2010.

16. Gibson JA, Hahn HP, Shahsafaei A and Odze RD: MUC expression in hyperplastic and serrated colonic polyps: Lack of specificity of MUC6. Am J Surg Pathol 35: 742-749, 2011.
17. Walsh MD,Clendenning M, Williamson E, Pearson SA, Walters RJ, Nagler B, Packenas D, Win AK, Hopper JL, Jenkins MA, et al: Expression of MUC2, MUC5AC, MUC5B, and MUC6 mucins in colorectal cancers and their association with the $\mathrm{CpG}$ island methylator phenotype. Mod Pathol 26: 1642-1656, 2013.

18. Kim J, Kim MA, Jee CD, Jung EJ and Kim WH: Reduced expression and homozygous deletion of annexin A10 in gastric carcinoma. Int J Cancer 125: 1842-1850, 2009.

19. Kim JK, Kim PJ, Jung KH, Noh JH, Eun JW, Bae HJ, Xie HJ, Shan JM, Ping WY, Park WS, et al: Decreased expression of Annexin A10 in gastric cancer and its overexpression in tumor cell growth suppression. Oncol Rep 24: 607-612, 2010.

20. Brooke NM, Garcia-Fernàndez J and Holland PW: The ParaHox gene cluster is an evolutionary sister of the Hox gene cluster. Nature 392: 920-922, 1998

21. Koizumi M, Doi R, Toyoda E, Masui T, Tulachan SS, Kawaguchi Y, Fujimoto K, Gittes GK and Imamura M: Increased PDX-1 expression is associated with outcome in patients with pancreatic cancer. Surgery 134: 260-266, 2003.

22. Ma J, Chen M, Wang J, Xia HH, Zhu S, Liang Y, Gu Q, Qiao L, Dai Y, Zou B, et al: Pancreatic duodenal homeobox-1 (PDX1) functions as a tumor suppressor in gastric cancer. Carcinogenesis 29: 1327-1333, 2008.

23. Sakamoto N, Feng Y, Stolfi C, Kurosu Y, Green M, Lin J, Green ME, Sentani K, Yasui W, McMahon M, et al: BRAFV600E cooperates with CDX2 inactivation to promote serrated colorectal tumorigenesis. Elife 6: e20331, 2017.

24. Yanagihara K, Tanaka H, Takigahira M, Ino Y, Yamaguchi Y, Toge T, Sugano K and Hirohashi S: Establishment of two cell lines from human gastric scirrhous carcinoma that possess the potential to metastasize spontaneously in nude mice. Cancer Sci 95: 575-582, 2004

25. Yasui W, Ayhan A, Kitadai Y, Nishimura K, Yokozaki H, Ito $\mathrm{H}$ and Tahara E: Increased expression of p34cdc2 and its kinase activity in human gastric and colonic carcinomas. Int J Cancer 53: 36-41, 1993.

26. Sakamoto N, Naito Y, Oue N, Sentani K, Uraoka N, Oo HZ, Yanagihara K, Aoyagi K, Sasaki H and Yasui W: MicroRNA-148a is downregulated in gastric cancer, targets MMP7, and indicates tumor invasiveness and poor prognosis. Cancer Sci 105: 236-243, 2014.

27. Yui S, Nakamura T, Sato T, Nemoto Y, Mizutani T, Zheng X, Ichinose S, Nagaishi T, Okamoto R, Tsuchiya K, et al: Functional engraftment of colon epithelium expanded in vitro from a single adult Lgr5 ${ }^{+}$stem cell. Nat Med 18: 618-623, 2012.

28. Koo BK, Sasselli V and Clevers H: Retroviral gene expression control in primary organoid cultures. Curr Protoc Stem Cell Biol 27: Unit 5A.6, 2013.

29. Livak KJ and Schmittgen TD: Analysis of relative gene expression data using real-time quantitative PCR and the 2(-Delta Delta C(T)) method. Methods 25: 402-408, 2001.

30. Kim TH and Shivdasani RA: Stomach development, stem cells and disease. Development 143: 554-565, 2016.

31. Sato T, Vries RG, Snippert HJ, van de Wetering M, Barker N, Stange DE, van Es JH, Abo A, Kujala P, Peters PJ and Clevers H: Single Lgr5 stem cells build crypt-villus structures in vitro without a mesenchymal niche. Nature 459: 262-265, 2009.

32. Fujii M, Shimokawa M, Date S, Takano A, Matano M, Nanki K, Ohta Y, Toshimitsu K, Nakazato Y, Kawasaki K, et al: A colorectal tumor organoid library demonstrates progressive loss of niche factor requirements during tumorigenesis. Cell Stem Cell 18: 827-838, 2016.

33. Bartfeld S, Bayram T, van de Wetering M, Huch M, Begthel H, Kujala P, Vries R, Peters PJ and Clevers H: In vitro expansion of human gastric epithelial stem cells and their responses to bacterial infection. Gastroenterology 148: 126-136.e126, 2015.

34. Cancer Genome Atlas Research Network: Comprehensive molecular characterization of gastric adenocarcinoma. Nature 513: 202-209, 2014.

35. van de Wetering M, Francies HE, Francis JM, Bounova G, Iorio F, Pronk A, van Houdt W, van Gorp J, Taylor-Weiner A, Kester L, et al: Prospective derivation of a living organoid biobank of colorectal cancer patients. Cell 161: 933-945, 2015. 